\title{
Vigorexia em praticantes masculinos do treinamento de força entre 18-35 anos
}

\author{
Vigorexia in male strength training practitioners \\ between 18-35 years
}

Fernando Sérgio dos Santos da Cunha Oliveira ${ }^{1}$

Celso Luiz Mastrascusa'

\section{RESUMO}

O presente estudo teórico buscou aferir a incidência da Vigorexia entre praticantes masculinos do treinamento de força entre 18-35 anos. Nos últimos anos foi diagnosticado um transtorno majoritariamente associado ao sexo masculino chamado Dismorfia Muscular ou Vigorexia que, entre outros aspectos, perturba a vida social, física, mental e psicológica do indivíduo. Essa síndrome caracteriza-se por um quadro onde o indivíduo se vê com um corpo fraco, franzino e pequeno, mas que na verdade não corresponde à realidade, pois sua percepção visual está equivocada. Na verdade, ele é portador de um corpo musculoso, volumoso. Assim, este transtorno se caracteriza por uma exacerbada preocupação em ter o corpo com a maior massa muscular possível. Esta síndrome acomete principalmente os homens jovens, entre 18-35 anos, que buscam no treinamento de força uma "arma" para alcançar o corpo sonhado. A Vigorexia tem muitas causas e ainda não todas esclarecidas, mas é universalmente aceite pelos autores a extraordinária influência da mídia sobre este novo conceito de biótipo físico masculino. Os jovens buscam uma afirmação social através do corpo. Para tal, utilizam substâncias perigosas para a sua saúde como os esteroides anabolizantes. Ao mesmo tempo praticam sessões de treinamento exaustivas na ânsia de obterem músculos maiores levando o corpo à exaustão, além de prejudicarem ossos, ligamentos, tendões e músculos. Outro aspecto muito importante deste transtorno é o psicológico do indivíduo, muito alterado e frequentemente ansioso, principalmente quando associado à utilização de esteroides anabolizantes. Conclui-se no estudo que o problema é crescente e já com uma incidência respeitável e merecedora da atenção dos órgãos públicos para combater este problema.

\section{PALAVRAS-CHAVE}

Dismorfia muscular; Imagem corporal; Treinamento de força; Mídia; Esteroide anabolizante.

1 Centro Universitário Metodista - IPA. 


\section{ABSTRACT}

The present theoretical study sought to assess the incidence of Vigorexia among male practitioners of strength training between 18-35 years. In recent years it has been diagnosed a mostly male-related disorder called Muscular dysmorphia or Vigorexia that, among other aspects, disturbs the social, physical, mental and psychological life of the individual. This syndrome is characterized by a picture where the individual sees himself with a weak, small and tiny body, but that does not actually correspond to reality, because his visual perception is wrong. In fact, he's carrying a massive, muscular body. Thus, this disorder is characterized by an exacerbated concern to have the body with as much muscle mass as possible. This syndrome mainly affects young men, between 18-35 years, who seek in the training of force a "weapon" to reach the body dreamed. The Vigorexia has many causes and not yet all clarified, but it is universally accepted by the authors the extraordinary influence of the media on this new concept of male physical biotype. Young people seek a social affirmation through the body. They use hazardous substances for their health, such as anabolic steroids. At the same time they practice extensive training sessions in the desire to get larger muscles leading the body to exhaustion, besides damaging bones, ligaments, tendons and muscles. Another very important aspect of this disorder is the psychological of the individual, very altered and often anxious, especially when associated with the use of anabolic steroids. It is concluded in the study that the problem is increasing and already with a respectable and worthy incidence of the attention of the public bodies to combat this problem.

\section{KEYWORDS}

Muscle dysmorphia; Body image; Strenght Training; Media. anabolic steroids. 


\section{INTRODUÇÃO}

Nos últimos anos alterou-se significativamente a forma como se entende o próprio corpo. A imagem que se tem de si mesmo é, na verdade, uma figuração corporal construída pela nossa mente (SCHILDER, 1999).

O conceito de Imagem Corporal é traduzido por uma estrutura multidimensional que nos revela a nossa própria aparência física assim como a comparação com a aparência dos outros (CASH et al., 1990). Tendo presente esse conceito pode afirmar-se que a Imagem Corporal (IC) é um componente cada vez mais imprescindível na própria identidade pessoal (QUADROS et al., 2010).

Ao longo das últimas décadas variadíssimos aspetos têm influenciado a mudança do conceito de Imagem Corporal: sexo, idade, meios de comunicação social (VEGGl et al., 2004). Além destes aspetos, também estão presentes fatores biológicos, psicológicos e socioculturais (ADAMS; KIRBY, 1998). As mudanças de conceito têm acontecido com a evolução da sociedade (BOSI et al., 2006) e tem gerado enormes aflições entre os indivíduos originando uma busca pelo corpo perfeito através, por exemplo, da prática da musculação (BLOWERS et al., 2003).

A Dismorfia Muscular, também conhecida como Vigorexia, traz preocupações que limitam as atividades diárias, seja através de uma prática excessiva de exercício físico ou num isolamento perante as atividades mais comuns da vida social. A obsessão com um corpo extraordinariamente musculoso potencializa sofrimento, angústia, baixa autoestima e confiança reduzida para uma vida social normal e integradora (MOTA; AGUIAR, 2011).

Enquanto que para as mulheres o tipo físico ideal é um corpo mais magro e menos volumoso, os homens ambicionam um corpo forte e musculoso, com baixo percentual de gordura (DAMASCENO et al., 2005). Os hábitos dos brasileiros estão mudando e os homens estão buscando o copo perfeito a qualquer preço. Isso inclui o uso de esteroides anabolizantes e dietas radicais, com alto teor de proteínas gerando transtornos alimentares e outros associados, tais como alterações de humor, ansiedade e comportamento (OLIVARDIA; POPE; HUDSON, 2000).
Perante esta nova realidade tem-se como objetivo do presente artigo aferir através de uma revisão teórica a incidência da dismorfia muscular em praticantes de força masculinos, com idades compreendidas entre os 18-35 anos.

\section{MATERIAIS E MÉTODOS}

Este estudo teórico narrativo foi realizado utilizando-se as bases de dados Google Scholar (Google Acadêmico), Scientific Eletronic Library Online (Scielo) e U.S.National Library of Medicine (PubMed) no período de 1990 até 2016. Também foram utilizados livros considerados relevantes na matéria estudada. A busca foi realizada em três idiomas: inglês, espanhol e português. Utilizaram-se como Keywords: Muscle Dysmorphia, Body Image, Power Training, Mídia and Anabolic Steroids e como palavras-chave: Dismorfia Muscular, Imagem Corporal, Treinamento de Força, Mídia e Esteroides Anabolizantes. Foi realizada uma pré-seleção de artigos nacionais (30 artigos) e internacionais (70 artigos) dos anos de 1990 a 2016. Desses foram utilizados doze (12) artigos nacionais e vinte e oito (28) artigos internacionais com os idiomas português, espanhol e inglês. Utilizaram-se como critérios de exclusão os artigos que não abordavam em sua temática a dismorfia muscular e cuja data de publicação fosse anterior a 1990.

\section{ATUALIZACÃO DA LITERATURA}

\subsection{ETIOLOGIA, CARACTERÍSTICAS E CAUSAS DA VIGOREXIA}

Ao longo dos tempos o percurso da humanidade foi-se moldando e a forma como os homens e as mulheres dignificaram o corpo demonstrou enorme irracionalidade. Destaca-se que em cada momento da história sempre afloraram determinados conceitos e padrões de beleza física. Assim, cada época promoveu o tipo de corpo ideal a ser seguido fazendo com que homens e mulheres buscassem essas referências como exemplos de sucesso, reconhecimento e admiração (FERREIRA et al., 2005). Atualmente ainda se busca aprofundar com mais clareza a etiologia da Vigorexia. Normalmente se buscam 
explicações em fatores potencialmente genéticos, outros que desencadeiam o transtorno e, por último, fatores que mantém o indivíduo naquela adição. Busca-se entender a inter-relação entre estes fatores e quais os tipos potencialmente existentes. Sabe-se que os fatores podem ser biológicos, psicológicos e socioculturais (ADAMS; KIRBY, 1998).

Relativamente à questão da exposição genética para potencializar o aparecimento deste transtorno certos autores apelidam de adição (MUNOZ; GOMEZ, 2004). Especula-se que um potencial menor rendimento do sistema relacionado com a produção do hormônio serotonina esteja presente neste tipo de transtorno. Além disso, pode existir uma menor capacidade de atuação considerada regular de outros neurotransmissores cerebrais, nomeadamente aqueles responsáveis pela inibição de comportamentos ou relativos à produção e síntese da dopamina, cujos níveis aumentam exponencialmente com a prática exacerbada de exercícios físicos (ALONSO, 2006). Outra linha de pensamento sobre esta questão afirma que o sistema nervoso fica dependente de um aumento da quantidade de catecolaminas inerentes à prática regular de exercícios físicos (MARTELL, 2006). Ainda segundo o autor isso aproximaria este transtorno de uma adição e não tão somente algo psicológico.

As variáveis socioculturais podem ser um "rastiIho de pólvora" desencadeando este processo. Sabe-se que os "media" promovem e instigam certos biótipos considerados modelos de sucesso. A exposição se dá desde criança interiorizando esta que tendo um corpo bonito tudo será fácil na vida adulta: sucesso profissional, social, com as mulheres, etc. No entanto, tudo isto só será possível se o seu corpo for esbelto, musculado e definido. Aliás, algo acessível, fácil de ser alcançado. Torna-se apenas necessário inscrever-se em uma academia e como que num golpe de mágica se obtém o corpo dos "sonhos". Só que a realidade é bem diferente e cruel. Rapidamente se apercebe que não é tão fácil como lhe fora prometido e instigado. Então, deverá treinar cada vez mais e mais. As consequências são uma diminuição da qualidade de vida, isolamento social, profissional e riscos para a sua própria saúde (FANJUL, 2006).

As questões psicológicas têm papel muito importante neste transtorno (DE LA PAZ, 2002; GARCIA,
2002). É muito comum os indivíduos sofrerem decepções relativas à sua aparência física, frustrações profissionais, incompreensões afetivas, entre outras questões. $O$ interessante é observar que o indivíduo passa a centralizar o sucesso de sua existência, ou seja, apenas terá um "DNA" vencedor se o seu físico for esbelto e reconhecido como sendo forte, másculo, atrativo. A busca pela perfeição física torna-se incessante, desafiadora, o único pensamento desfilando em seu cérebro (DE LA SERNA, 2004).

Os primeiros estudos científicos relevantes sobre a dependência do exercício físico aparecem descritos nos trabalhos de Baekeland (1970 apud ARBINAGA, 2004), nos quais se estudava o efeito da privação do exercício na qualidade do sono. Nos anos noventa começou-se a estudar o fenômeno no qual a prática desportiva se converte em una "obsessão". As primeiras referências são encontradas no grupo de investigação do psiquiatra Harrison Pope nos Estados Unidos, que descreveu a "doença" pela primeira vez em 1993 enquanto investigava o uso e abuso de esteroides (POPE, 1993; MUNOZ MARRÓN; GÓMEZ CAMPELO, 2003). Na realidade Popejá havia estudado antes este transtorno que inicialmente considerou como uma forma de psicose (POPE, 1987).

Os autores Harrison Pope e sua equipe (1993) mostraram relatos perturbadores de nove homens com vigorexia e após esse fato escreveram um artigo sobre o tema. Para destacar o fato resolveram mudar a terminação da síndrome apelidando-a de anorexia nervosa reversa. $\mathrm{O}$ artigo gerou uma grande discussão e provocou inúmeras reflexões, mostrando que a anorexia nervosa reversa não era tão inofensiva quanto se imaginava. Tempos depois, ainda na década de 90, a síndrome sofreu nova mudança de terminologia, dado que Pope Jr. comprovou que não derivava de um transtorno alimentar, mas pelo contrário, se tratava de um Transtorno Dismórfico Corporal (TDC), sendo chamada de dismorfia muscular. Esta se caracteriza por uma preocupação de não ser suficientemente forte e musculoso em todas as partes do corpo, ao contrário de outros transtornos corporais (TDC's), cuja principal preocupação é com áreas específicas (ASSUNÇÃO, 2002). A quantidade de tempo que dedicam ao exercício físico (mentalmente ou na prática) dificultando muitas vezes uma plena 
integração social, laboral, acadêmica e familiar (DE LA SERNA, 2004) e as alterações no conceito de imagem corporal, se achando fracas e com pouca hipertrofia muscular (BAILE, 2005; POPE, 1993) são as características principais que caracterizam a dismorfia muscular.

Segundo estudos de Cristiane Mota e Eduarda Aguiar (2011) a Vigorexia traz preocupações que limitam as atividades diárias, pois os indivíduos portadores desta síndrome acabam dedicando grande parte do seu tempo diário à prática de exercícios físicos, objetivando somente a hipertrofia muscular, ignorando muitas vezes os cuidados necessários com a sua própria saúde física e mental. Na vigorexia os indivíduos manifestam sinais de ansiedade evitando manifestações sociais por temerem críticas relativas à sua aparência física. A obsessão com a musculatura potencializa sofrimento, angústias e temores que Ihes impedem ter uma vida social normal e integradora. A aparência física tornou-se a identidade máxima para estes indivíduos, a forma de ser aceite, respeitado e admirado pelas outras pessoas.

As causas da vigorexia são diversas, desde traumas na infância até pensamentos e sentimentos reprimidos do próprio corpo. Segundo Pope, Phillips e Olivardia (2000, p. 284), "A exposição durante a infância a imagens corporais masculinas irreais- dos bonecos aos anúncios nas revistas, passando pelos programas de televisão - indubitavelmente contribuem para o Complexo de Adônis." Sabe-se que a construção da imagem corporal está intimamente ligada aos aspetos fisiológicos, emocionais e, principalmente, sociais. Para Cristiane Mota e Eduardo Aguiar (2011), os aspetos os aspetos socioculturais desempenham um papel importante na etiologia da dismorfia muscular, pois é notória a importância da aparência física para a sociedade atual. As pessoas devem entender que é importante promover saúde física e mental e não ser "escravo" de ideais de beleza artificiais e surreais promovidos pela mídia. A aparência física não deve ser alvo de uma obsessão relativamente à perfeição, pois o ser humano é muito mais do que um corpo (POPE; PHILLIPS; OLIVARDIA, 2000).

Para Sarah da Costa et al. (2010) não ter um corpo atlético significa preguiça, descuido, não possuir força de vontade para atingir objetivos. $O$ corpo pas- sou a ter uma importância social, dar "status", pois quem está obeso ou fora de forma é marginalizado, discriminado e colocado de parte por certos setores sociais. Se a pessoa tem uma aparência física nobre esta consegue o respeito e admiração dos outros possibilitando uma ascensão social relevante. O corpo passou a ser, efetivamente, um instrumento indispensável de reconhecimento por parte dos outros. Quem não possui um corpo atlético sente que é colocado de parte em certos ambientes sociais e, inclusive, é "vítima" de preconceito e comentários nada ou pouco abonatórios.

\subsection{INFLUÊNCIA DA MÍDIA}

Nos tempos modernos a mídia conquistou um poder de convencimento das pessoas muito forte. Estamos falando de veículos de comunicação poderosos com capacidade de promover valores que mexem com a vida cotidiana da sociedade onde estão inseridos. Televisão, mídia impressa, estações de rádio e internet têm capacidade de promover conceitos sobre todos os assuntos. Na questão relacionada com a aparência física estes veículos promovem determinados padrões estéticos completamente inacessíveis para a maioria das pessoas. Isso origina uma necessidade das pessoas em se adequar a esse padrão estético para não serem marginalizados, desprezados ou ignorados. A imagem corporal está cada vez mais intrinsecamente associada com a autoestima do indivíduo, potencializando o amor próprio, a satisfação pessoal e, sobretudo, ter uma relação positiva e tranquila consigo mesmo, com sua própria imagem. A autoconfiança é perdida quando não se reconhece no próprio corpo o estereótipo idealizado pela sociedade (BUCARETCHI, 2003).

As pessoas passam a ter uma relação de desprezo com o próprio corpo e buscam meios de obter o corpo "dito" perfeito e belo. Com o objetivo de atingir esse padrão estético as pessoas buscam utilizar todos os meios possíveis, muitas vezes prejudiciais à sua própria saúde, tais como dietas radicais, uso de fármacos, esteroides anabolizantes. A responsabilidade de não ter um corpo esteticamente perfeito é de responsabilidade o próprio indivíduo. Essa ideia dissemina o entendimento de que não existem des- 
culpas para não ser atraente, perfeito, atlético, pois existem infindáveis recursos e opções como cirurgias plásticas, dietas exercícios (Severiano, Rêgo e Montefusco, 2010). A auto avaliação da imagem corporal normalmente ocorre através de três formas: o indivíduo é muito crítico relativamente à sua aparência; compara a sua aparência com padrões extremos reverenciados socialmente; o indivíduo potencializa um aspecto de sua aparência (SAIKALI et al., 2004; CHOI; POPE; OLIVARDIA, 2002).

Os homens se reconhecem em corpos cada vez mais atléticos, fortes, musculosos. Eles acham que esse biótipo físico lhes trará reconhecimento, destaque, projeção social. Essa distorção da imagem parece enquadrar-se na rotina ao longo dos tempos da mulher em se adequar ao padrão corporal ideal. Nos dias atuais o homem busca o seu espaço na sociedade cada vez mais viril e segregadora, sendo o corpo o seu instrumento privilegiado de afirmação (ASSUNÇÂO, 2002). Na ânsia de obter um corpo musculoso os indivíduos portadores desta doença não medem esforços para alcança-lo. Provem rotinas diárias de treinamento exaustivo, dietas rigorosas e, inclusive, utilizam substâncias proibidas e muito danosas para a sua saúde. Com isso, alteram completamente suas vidas no aspecto social trazendo desconforto para as pessoas próximas. É um transtorno tipicamente masculino embora haja casos também entre algumas mulheres. As exigências exageradas e muitas vezes irracionais relativamente ao próprio corpo originam desde pequenos desconfortos com partes de seu corpo até obsessões devastadoras (POPE Jr.; PHILLIPS; OLIVARDIA, 2000).

A interação entre o que a indústria da mídia, apoiada e patrocinada por grandes corporações do ramo da estética, e a fantasia por ela estimulada na mente das pessoas provoca desejos, imagens de sucesso, fantasias relativamente à conquista do corpo perfeito. As pessoas passam a acreditar que tendo um corpo perfeito irão realizar todos os seus sonhos e desejos (RUSSO, 2005). De acordo com Severiano, Rêgo e Montefusco (2010), a responsabilidade de não ter um corpo esteticamente perfeito é de responsabilidade do próprio indivíduo. Essa ideia dissemina o entendimento de que não existem desculpas para não ser atraente, perfeito, atlético, pois existem infindáveis recursos e opções como cirurgias plásticas, dietas e exercícios. Para isso é necessário incorporar os estereótipos de beleza ditados pela mídia. Desse modo a beleza torna-se acessível, igualitária, artificializada, passível de ser adquirida sem esforço. Inclusive, não existe mais o monopólio da beleza por um restrito estrato social (classe $A$ ) dado que todos podem ter acesso a esse padrão de beleza (IRIART et al., 2009).

Segundo Falcão (2008), estamos vivendo um tempo cuja ênfase é dada ao corpo perfeito cujas características se resumem a um homem musculoso, atlético, vencedor. Este processo vivido pelos homens nos últimos anos se aproxima do vivenciado e exigido às mulheres há décadas. A necessidade de possuir um corpo perfeito segundo os padrões ditados na atualidade provocam inúmeras assimetrias com o que se verifica diante do espelho. Esta profunda distorção entre a realidade e o que se vê no espelho é sintomática da presença do transtorno dismórfico corporal (FERREIRA; CASTRO; GOMES, 2005). Para Falcão (2008), existe uma correlação entre à Dismorfia Muscular e outras doenças, nomeadamente a Dependência de Exercícios Físicos e o Abuso de Substâncias. Culturalmente o homem possui uma menor capacidade de expor suas angústias, desejos, inquietações, medos, receios, indecisões, fraquezas. Dessa forma, a Dismorfia Muscular engloba-se nas patologias difíceis de diagnosticar, prevenir e tratar.

Este transtorno provoca inúmeros pensamentos obsessivos e comportamentos sistematizados relativamente à rotina diária dos portadores de Dismorfia Muscular, nomeadamente quanto às questões de treinamento e dieta alimentar (PORTO; LINS, 2009). Para muitos jovens a tentativa de alcançarem um corpo exacerbadamente atlético aumentará a sua capacidade de atrair um parceiro sexual. Já para outros homens, isso é fruto de uma "masculinidade ameaçada". Isso é fruto de uma necessidade de afirmação dentro de um contexto, grupo social. É algo histórico ao longo dos tempos de que os homens másculos atraem olhares de admiração, respeito e cobiça (POPE; PHLILLIPS; OLIVARDIA, 2000). 


\subsection{GRUPOS DE RISCO}

Os indivíduos portadores da Vigorexia usualmente afirmam estar com o corpo "fraco e pequeno", quando na verdade possuem uma musculatura muito volumosa em níveis muito acima da verificada pela média da população masculina, manifestando uma óbvia e preocupante distorção da própria imagem corporal. Estes indivíduos vivem obsecados com o tamanho de sua massa muscular, o que pode potencializar problemas físicos e psicológicos devido ao excesso de levantamento de peso, prática de dietas rigorosas e perigosas assim como o uso sem orientação médica de suplementos proteicos, além do consumo de esteroides anabolizantes (GUARIN, 2002; CAFRI; VAN DEN BERG; THOMPSON, 2006; GRIEVE, 2007, HILDEBRAND et al., 2006).

Estes indivíduos evitam eventos sociais onde necessitam expor seus corpos em público, já que sentem vergonha. Para tal, usam roupas inapropriadas para o local ou época do ano com intuito de evitar esta exposição (ASSUNÇÃO, 2002; CHOI; POPE; OLIVARDIA, 2002; CHUNG, 2001; OLIVARDIA; POPE; HUDSON, 2000). Atualmente, o indivíduo interiorizou que somente será aceite pelo grupo social se apresentar um biótipo físico semelhante com os do grupo (MELIN; ARAÚJO, 2002). Assim, pessoas consideradas sem um corpo atrativo imediatamente são discriminadas e desencorajadas a pertencerem a tal grupo. Esta postura social potencializa a ausência de habilidades sociais e provoca baixa autoestima nos indivíduos (SAIKALI et al., 2004).

A maior incidência da Vigorexia ocorre entre homens dos 18 aos 35 anos sendo expressa por fatores socioeconômicos, emocionais, fisiológicos, cognitivos e comportamentais. O nível socioeconômico dos portadores deste transtorno é diverso, mas é mais frequente na classe média baixa (ALONSO, 2006; GRIEVE, 2007). A obsessão pela prática de atividades físicas resulta como que num fanatismo religioso, colocando à prova constantemente os limites do indivíduo, sua forma física apresentando consequências potencialmente gravosas para o seu organismo (ALONSO, 2006).

Outro aspecto importante é o fato de que potenciais problemas nos corpos destes indivíduos passa- riam despercebidos para a maioria das pessoas. No entanto, estes problemas se apresentam como reais para estas pessoas, originando depressão, ansiedade, problemas no trabalho e nas relações sociais (ALONSO, 2006; CHUNG, 2001; LEONE; SEDORY; GRAY, 2005; OLIVARDIA; POPE; HUDSON, 2000; POPE et al., 1997).

Para o autor Baptista (2005), os praticantes do treinamento de força em academias que praticam exercício físico em excesso na busca de um corpo perfeito, fazem parte de um potencial grupo de portadores do transtorno da Vigorexia. Esses indivíduos buscam o perfeccionismo físico passando horas diárias realizando rotinas de treinamento. Esta obsessão pela busca incessante da beleza física pode originar ansiedade, depressão, fobias, atitudes compulsivas e repetitivas - como constantes poses diante do espeIho (ASSUNÇÃO, 2002).

Um estudo realizado por Lantz, Rhea e Cornelius (2002), com 100 fisiculturistas profissionais mostrou que o grupo apresentou elevadas porcentagens de indicadores de Vigorexia, principalmente preocupação com tamanho dos músculos e respectiva simetria. Isto indica que fisiculturistas poderá ser um grupo de risco para a manifestação deste transtorno. Em outro estudo realizado na África do Sul por Hitzeroth et al. (2001), com 28 fisiculturistas amadores apresentou-se uma prevalência de 53,6\% de Vigorexia.

\subsection{VIGOREXIA E O USO DE ESTEROIDES ANABOLIZANTES}

O homem moderno na faixa etária dos 18-35 anos assume o próprio corpo como a principal forma de expressão no seu meio social (AGUIR, 2003). Assim, tendo por objetivo alcançar o corpo sonhado e difundido como belo e perfeito pela mídia, estes indivíduos utilizam substâncias para potencializar os resultados do treinamento de força em sua musculatura (SANTOS e SANTOS, 2002).

A busca por todos os recursos possíveis para aumentar a massa muscular, nomeadamente o uso de esteroides anabolizantes, aumenta os riscos de problemas sérios na saúde dos indivíduos, colocando muitas vezes em risco sua própria vida (ABRAHIN e SOUSA, 2013). Além disso, fruto do transtorno da 
Vigorexia, eles se veem fracos, sem vigor, com musculaturas de pequeno porte quando na verdade já apresentam musculaturas muito desenvolvidas e atléticas (SOLER et al., 2013).

Os autores Olivardia, Pope e seus colaboradores (2000) realizaram um estudo onde foi possível comparar portadores do transtorno de Vigorexia com atletas levantadores de peso que não apresentavam o transtorno referido. No estudo realizou-se a comparação entre 24 homens com Vigorexia e 30 levantadores de peso normais. Aqueles que apresentavam sintomas da Vigorexia mostraram-se muito mais insatisfeitos com a imagem corporal, faziam uso de esteroides anabolizantes e eram mais acometidos por mudanças de humor, ansiedade e distúrbios alimentares ao longo da vida.

No período da Segunda Guerra Mundial (19391945) registrou-se a utilização da prática do uso de esteroides anabolizantes pelas tropas alemãs com o objetivo de aumentar sua agressividade e força. Trata-se do primeiro registro oficial do uso de esteroides anabolizantes com o intuito de aumentar o desempenho físico (DICKERMAN et. al.,1995). Em meados de 1954, a prática do uso de esteroides anabolizantes foi detectada em atletas russos de ambos os sexos. Os portadores do transtorno da Vigorexia têm mais predisposição para a utilização de esteroides anabolizantes já que buscam incessantemente aumentar o volume de seus músculos (CAFRI; VAN DEN BERG; THOMPSON, 2006; GUARIN, 2002).

Os esteroides androgênicos anabolizantes são derivados sintéticos do hormônio masculino testosterona que promovem o crescimento celular e auxiliam o desempenho de atletas. O seu uso potencializa um incremento na capacidade óssea e muscular. Eles podem ser ministrados por via oral e/ou injetável. O seu uso acarreta situações graves para o organismo e está associado ao surgimento de problemas físicos, cognitivos e psiquiátricos. Os principais problemas associados à utilização de esteroides anabolizantes dizem respeito às doenças coronarianas, hipertensão arterial, tumores hepáticos, hipertrofia prostática, hipogonadismo, problemas de ereção, atrofia testicular, atrofia mamária, alteração da voz, hipertrofia do clitóris e amenorreia em mulheres, aumento da força, produção de eritropoietina e glóbu- los vermelhos, formação óssea e a quantidade de massa magra, aumento do desejo sexual, ocorrência de acne, aumento na incidência de aterosclerose e aumento da quantidade de pelos corporais. Relativamente às alterações psiquiátricas pode-se destacar o incremento de comportamentos agressivos, hipomania e quadros psicóticos (ASSUNÇÃO, 2002; HARTGENS; KUIPERS, 2004).

Para Alonso (2006) a ingestão de esteroides anabolizantes pode provocar enormes danos inclusive à massa muscular em longo prazo, pois após a adaptação inicial o corpo demandará cada vez mais a necessidade do uso dessas drogas artificiais. Eles aumentam a atividade orgânica e processual do sistema nervoso central, do músculo liso e esquelético. Como os portadores de Vigorexia realizam sessões contínuas e exaustivas de treinamento físico acabam por liberar enormes quantidades do hormônio endorfina. Com isso, aumenta de uma forma exponencial a sensação de prazer escondendo e camuflando dor, cansaço, exaustão física. Ainda segundo o autor em longo prazo isso é muito prejudicial, pois sujeita o corpo a um stress muito violento. Desse modo os acometidos pela Vigorexia realizam cada vez mais sessões de treinamento em busca do bem estar, não tendo consciência plena dos riscos que para a sua saúde.

Outro aspecto muito importante nos vigoréxicos é a sobrecarga utilizada nas sessões de treinamento. $\mathrm{Na}$ ânsia de obter mais massa muscular colocam enormes cargas de resistência acarretando danos aos ossos, tendões, músculos e articulações, principalmente dos membros inferiores. É muito importante conscientizar essas pessoas para os perigos deste transtorno (ALONSO, 2006).

\subsection{INCIDÊNCIA DA VIGOREXIA ENTRE PRATICANTES MASCULINOS DO TREINAMENTO DE FORÇA}

Nos últimos anos assiste-se a um incremento na demanda do treinamento de força nas academias. A sociedade exige cada vez mais perfeição física e o treinamento de força é o método mais usado na busca por um biótipo musculoso, definido, atlético. Cada vez mais as pessoas estão tentando afirmação social através do corpo e isso também é fruto da 
pressão da mídia, como vimos anteriormente. Posto isso, os indivíduos procuram se encaixar no corpo "perfeito" e para isso adotam variadíssimas estratégias de treinamento, nomeadamente o treinamento de força. A imagem corporal, os transtornos relacionados a ela, bem como o uso de drogas relacionadas ao corpo são aspectos muito penetrantes na sociedade atual, nomeadamente entre os indivíduos jovens do sexo masculino (RUSSO, 2005).

$\mathrm{Na}$ ânsia de obter a imagem corporal perfeita, para ser reconhecido pela sociedade como belo, os indivíduos ultrapassam barreiras inacreditáveis desrespeitando a diversidade individual, nomeadamente a etnia e a cultura do meio a que pertence (ALVES et al., 2009). Assiste-se a uma brutal homogeneização do padrão estético, sendo que as pessoas se "aprisionam" dentro de modelos estereotipados. Assim, os indivíduos iniciam uma busca incessante pelo corpo idealizado nos tempos atuais. O perfeito hoje é ser magro para as mulheres e musculoso para os homens (ALVARENGA et al., 2010). No entanto, esta busca origina diversos transtornos associados a esta desconstrução da imagem corporal, sendo a anorexia a mais comum nas mulheres e a vigorexia em homens (SAIKALI et al., 2004). A percepção que se tem do corpo se entende por imagem corporal, isto é, é a realização mental das imagens obtidas da visualização do próprio corpo (SCHILDER, 1999).

Assim, pode observar-se que a mente cria "ilusões" não reais acerca do tamanho dos músculos provocando respostas emocionais condizentes com a imagem criada na mente (QUADROS et al., 2010). Essa imagem recorrentemente promove distorções graves, quando comparada com a realidade. A percepção da própria imagem potencializa insatisfações de ordem mental no indivíduo, com consequências graves de ordem psicológica (FREIRE, 2013). A exagerada obsessão por um corpo escultural demanda cada vez mais recursos para atingi-lo, muitas vezes inalcançável. Com isso, são geradas muitas insatisfações provocando o aparecimento de transtornos relacionados com a imagem corporal, nomeadamente a vigorexia e anorexia (PEREIRA, 2009). Dessa forma a imagem corporal é um atributo fundamental e primordial da própria identidade (QUADROS et al., 2010).
Estudos recentes revelam um incremento acentuado deste transtorno nos indivíduos praticantes do treinamento de força (DERENNE; BERESIN, 2006). Os brasileiros vêm evidenciando mudanças corporais, dado que as exigências atuais demandadas pela mídia exacerbaram a necessidade de estar em excelente condição física (SILVA; GOMES, 2008). Entre estas rápidas mudanças interessa para este trabalho destacar o crescimento desenfreado, sobretudo nas sociedades mais desenvolvidas, as chamadas "novas adições". Estas "adições sem utilização de droga" surgem como uma atividade, aparentemente inócua, que realiza o sujeito de maneira repetitiva e que lhe oferece satisfação e prazer, possibilidade de atingir determinado objetivo e uma extraordinária sensação de controle das situações (MUNOZ MARRÒN; GÓMEZ; CAMPELO, 2003).

Por outro lado, é importante destacar a existência do culto excessivo da beleza corporal e uma obsessão por um biótipo entendido como perfeito. O corpo transformou-se para inúmeras pessoas no aspecto mais transcendente de sua identidade e no referente máximo de sua afirmação e valorização no interior de seu grupo social. Os meios de comunicação social promovem insistentemente, através de filmes e publicidade, que uma pessoa feliz, saudável é aquela que tem um corpo bonito. Ainda segundo Olivardia et al. (2004) a maioria dos norte-americanos estão descontentes com o seu corpo e esse fato provoca índices elevados de autoestima reduzida, busca por substâncias perigosas, mudanças radicais na dieta alimentar e depressão. A necessidade de um corpo perfeito resulta variadíssimas vezes em diversos transtornos psicológicos (YARYURA-TÓBIAS, 2003).

Esta temática é de difícil aferição dado existir pouquíssimos estudos quantitativos. Torna-se necessário, aliás, produzir uma quantidade maior de pesquisas quantitativas para que se possa ter uma ideia mais concreta e precisa acerca da prevalência e incidência da Vigorexia em praticantes do treinamento de força com idades compreendidas entre os 18-35 anos. Os estudos quantitativos existentes são mais generalistas, mas servem de base para este estudo de revisão teórica.

O autor Olivardia (2001) referenciou em seu estudo que noventa mil (90000) americanos demons- 
travam sofrer do transtorno da Vigorexia. Outro estudo realizado no México (BAILE, 2005) revelou um percentual semelhante entre a população masculina do país. Ainda segundo o mesmo autor, agora em um estudo realizado na Espanha, no ano de 2005, revelou que cerca de dez mil e quinhentos (10500) homens eram vigoréxicos. Pope e seus colaboradores (2000) concluíram que o problema da Vigorexia é comum a inúmeras culturas.

Outros autores reconhecidamente atuantes na matéria produziram estudos relevantes para o descortino da dimensão do problema. Assunção (2002) afirmou que a Vigorexia é um problema basicamente pertencente ao universo masculino. Os autores Leone, Sedory e Gray (2005) afirmaram em seu estudo que a incidência da Vigorexia está aumentando nos Estados Unidos da América assim como em outras regiões do mundo. No Brasil, os autores Pinheiro e Giugliani (2006), Bosi e colaboradores (2008) e Quadros (2010) concluíram que entre $17.4 \%$ a $82 \%$ da população não esteja contente e realizada com sua imagem corporal.

Destaque para os estudos específicos com fisiculturistas realizados por Lantz, Rhea e Cornelius (2002) e Hitzeroth e seus colaboradores (2001). O primeiro estudo envolveu a pesquisa com cem (100) fisiculturistas profissionais revelando uma taxa superior a $50 \%$ do transtorno da Vigorexia. Já o segundo estudo realizado com vinte e oito (28) fisiculturistas amadores aferiu uma prevalência de 53,6\% do transtorno da Vigorexia.

Por último, referência para alguns estudos realizados nos últimos anos no Brasil. O estudo realizado por Fred Zimmermann afirma que $23 \%$ dos praticantes jovens adultos do treino de força em Biguaçu, Santa Catarina, apresentavam sintomas de vigorexia. Em outro estudo realizado por Sant Ana e Teixeira Arroyo (2001) na cidade de Bebedouro, São Paulo, o percentual de portadores de vigorexia em praticantes adultos jovens de musculação atingiu $44 \%$. $O$ autor Chotao (2011) realizou um estudo em Curitiba, Paraná, onde foi aferido que $37.5 \%$ dos praticantes do treinamento de força com idades compreendidas entre os 18-35 anos foram diagnosticados como sendo portadores de vigorexia.

\section{CONCLUSÃO}

Este Estudo Bibliográfico teve como principal objetivo esclarecer o conceito de vigorexia e sua incidência entre jovens do sexo masculino praticantes do treinamento de força, mediante a revisão bibliográfica d artigos relacionados com o tema. Ainda que, atualmente, haja uma maior quantidade de literatura disponível se observa uma enorme ausência de dados científicos verossímeis, principalmente dados quantitativos.

As questões apresentadas com maior relevância estão relacionadas com as possíveis origens, causas e consequências desse transtorno do mundo pós-moderno denominado Dismorfia Muscular, Vigorexia ou Complexo de Adônis. Ais do que a nomenclatura usada para diagnosticar esse transtorno de imagem, o $x$-factor, ou seja, o aspecto transcendente que decisivamente marca este tipo de transtorno é a ressignificação do olhar sobre o corpo construído e transformado que tais condições patológicas apresentam.

A influência mediática cada vez mais forte, decisiva no formular dos conceitos de comportamento social, decorrente das novas tecnologias disponíveis e das transformações sociais da sociedade atual, apresenta-se como o veículo mais poderoso para propagar e disseminar uma desastrosa noção de padrão estético, moderno, "fashion", ideal. É inequívoco que a sociedade atual está propensa às imposições culturais que são impostas, formuladas, propagadas e transmitidas pelos meios de comunicação social.

A sociedade atual objetiva a idealização de biótipos perfeitos que devem ser almejados e conquistados como forma de reconhecimento social, prestígio e admiração por todos os outros que o rodeiam. As pessoas buscam no corpo a afirmação social que, variadíssimas vezes, não a conseguem alcançar por outros meios. A transformação dos valores sociais vivenciados pelas novas gerações apresenta o corpo musculado como o primeiro objetivo a ser alcançado com a promessa de trazer felicidade, realização, afirmação social. O sentimento vivenciado por sentir-se belo e admirado estimula as pessoas a tentar alcança-lo, ainda que com consequências físicas, psicológicas e sociais nefastas e preocupantes. 
Em períodos distintos da história da humanidade as questões relacionadas com o corpo foram debatidas de forma diferente e com abordagem própria da época estudada. O homem passou a rivalizar com a mulher nas questões estéticas, ou seja, vivemos tempos onde não somente a mulher é cobrada por sua aparência corporal. Assiste-se a um culto corporal masculino intenso por corpos atléticos, imponentes, gerando uma pressão social para esta realização. Torna-se necessário analisar esta transformação de padrões estéticos masculinos através de uma olhar clínico, social, psicológico, científico, antropológico, pedagógico, fisiológico e anatômico.

Os meios usados para alcançar este padrão de beleza extraordinário levantam sérias questões éticas e médicas. Aspetos bioenergéticos, suplementação agressiva, dietas rigorosas, uso de esteroides anabolizantes e superação de limites para além do saudável demandam reflexões urgentes sobre a matéria. Todos estes aspetos necessitam ser fortemente avaliados e discriminados para que se possam evitar riscos gravíssimos para a saúde dos homens, nomeadamente os mais expostos que são os jovens entre os 18-35 anos, praticantes do treinamento de força.

É muito preocupante o conceito de que todos os homens podem possuir corpos atléticos, musculosos. Com isso, passa-se a responsabilidade de atingir esse padrão pré-estabelecido para o indivíduo, ou seja, quem não consegue enquadrar-se nesse perfil é fraco, um "perdedor". As pessoas que não possuem um corpo atlético sentem-se excluídos, inferiores, marginalizados por certos setores sociais. Isto levanta muitos questionamentos, reflexões e análises fazendo com que o profissional de educação física tenha a capacidade de influenciar positivamente as outras pessoas, através do seu conhecimento científico sobre a matéria.

Conclui-se que a vigorexia tem uma incidência crescente e preocupante entre os jovens do sexo masculino praticantes do treinamento de força. Para um melhor esclarecimento de todas as varáveis envolvidas na questão recomendam-se mais estudos de pesquisa. No entanto, os estudos deverão incluir o entorno social do indivíduo portador da Dismorfia Muscular, vulgo Vigorexia, pois todas as pessoas que convivem diariamente com ele acabam por sofrer as consequências e transtornos inerentes a esta doença. Apesar de pouquíssimos estudos quantitativos sobre a matéria é aceite pelos pesquisadores um universo de afetados por este transtorno na faixa dos $4 \%$ aos $10 \%$ da população masculina com idade inferior aos 35 anos, sendo que nos praticantes do treinamento de força o percentual poderá ser ainda mais substancial. Assim, se juntarmos os portadores com o seu núcleo familiar e/ou social básico observa-se um número francamente elevado dos indivíduos afetados direta e/ou indiretamente por este problema, afetando extraordinariamente as relações e hábitos sociais.

\section{REFERÊNCIAS}

ABRAHIN, O.S.C. et al. Prevalência do uso e conhecimento de esteroides anabolizantes androgênicos por estudantes e professores de educação física que atuam em academias de ginástica. Rev Bras Med Esporte, v19, Belém - PA, 2013.

ADAMS, J.; KIRBY, ROBERT, J. El exceso de ejercicio como adicción. Una revisió. Revista de toxicomanías, v. 34, p. 10-22, 2003

AGUIAR, EDUARDO. O papel do stress nos processos de comunicação do corpo. Dissertação (Mestrado em Comunicação) - Departamento de Pós- Graduação em Comunicação e Semiótica da Pontifícia Universidade Católica de São Paulo, SP, 2003.

ALONSO, J. M. La adicción al gimnasio: Vigorexia o Complejo de Adonis. Madrid, 2006.

ALVARENGA, M. S. et al. Insatisfação com a imagem corporal em universitárias brasileiras. J. bras. psiquiatr., Rio de Janeiro, v. 59, n. 1, p. 44-51, 2010.

ALVES, D. et al. Cultura e imagem corporal. Rev. Motricidade, v. 5, n.1, p. 1-20, 2005.

ARBINAGA, F. Dependencia del ejercicio. Medicina Psicosomática y Psiquiatríade enlace, v. 71/72, p. 24-32, 2004. ASSUNÇÃO, S. S. M. Dismorfia Muscular. Rev. Bras. Psiquiatr., São Paulo, v. 24, suppl. 3, 2002.

BACURAU, R.F. Nutrição e Suplementação Esportivas. 5. ed. São Paulo: Phorte, 2007.

BAECHLE, T.R.; GROVES, B.R. Treinamento de força: passos para o sucesso. Porto Alegre: Artmed, 2000.

BAILE, J. I. Vigorexia: Cómo reconocerla y evitarla. Síntesis. Madrid, 2005. 
BAPTISTA, A. N. Distúrbios alimentares em frequentadores de academia. Revista Digital Buenos Aires., v. 10, n. 82, 2005.

BLOWERS, L. C. et al. The relationship between sociocultural pressure to be thin and body dissatisfaction in preadolescent girls. Eating Behaviors., v. 4, p. 229-244, 2003.

BOSI, M. L. M. et al. Autopercepção da imagem corporal entre estudantes de nutrição: um estudo no município do Rio de Janeiro. J Bras Psiquiatr., v. 55, n. 2, p. 108-113, 2006.

BUCARETCHI, H. A. Anorexia e Bulimia Nervosa uma visão multidisciplinar. São Paulo: Casa do psicólogo, 2003.

CAFRI, G.; VAN DER BERGH, P.; THOMPSON, J.K. Pursuit of muscularity in adolescent boys: relations among biopsychosocial variables and clinical outcomes. J Clin Child Adolesc Phychol, 2006.

CASH, T. F. et al. A. Body images: development, deviance and change. New York: The Guilford Press. 1990.

CHOI, P. Y. L.; POPE, H. G.; OLIVARDIA, R. Muscle Dysmorphia: a new syndrome in weightlifters. Br J Sports Med, v. 36, n. 5, p. 375-6, 2001.

CHOTÃO, S.L. A incidência de vigorexia em alunos de musculação de academias de Curitiba. Monografia apresentada ao curso de Educação Física da UTP. Paraná, 2001. CHUNG, B. Muscle dysmorphia: A Critical Review of the Proposed Criteria. Perspectives in Biology and Medicine, 2001. Disponível em http://www.ncbi.nlm.nih.gov/pubmed/11600802

COSTA, SARAH et al.. Distúrbios da imagem corporal e transtornos alimentares em atletas e praticantes de atividade física. Revista Digital Buenos Aires, n144, São Camilo, 2007.

DAMASCENO, V. O. et al. Tipo Físico Ideal e Satisfação com a Imagem Corporal de Praticantes de Caminhada. Revista Brasileira Medicina do Esporte, v. 11, n. 3. 2005.

DE LA PAZ, F. Vigorexia: un nuevo transtorno mental que afecta especialmente a hombres, 2002.

DE LA SERNA, I. Alteraciones de la imagen corporal: Anorexia, Vigorexia, Bulimia, Dismorfofobia y cuadros relacionados. Monografías de Psiquiatría, v. 2, n. 16, p. 32-40, 2004.

DERENNE, J. L.; BERESIN, E. V. Body Image, Media, and Eating Disorders. Academic Psychiatry. Academic Psychiatry, 2006.

DICKERMAN, R. D. et al. Sudden cardiac death in a 20-yearold bodybuilder using anabolic steroids. Cardiology, v. 86, n. 2, p. 172-173, 1995.
FALCÃO, R. S. Interfaces entre dismorfia muscular e psicologia esportiva. Revista Brasileira de Psicologia do Esporte, São Paulo, v. 2, n. 1, p. 1-21, 2008.

FANJUL, C. La apariencia y características físicas de los modelos publicitarios: Códigos no verbales de la realidad en el discurso publicitario como factor de influencia social mediática en la vigorexia masculina. Tesis Doctoral, Universidad Jaume I, Castellón, 2006.

FERREIRA, M. E. C.; CASTRO, A. P. A.; GOMES, G. A obsessão masculina pelocorpo: malhado, forte e sarado. Rev. Bras. Cienc. Esporte, Campinas, v. 27, n.1, p. 167-182, 2005.

FREIRE, L. A. Bases Valorativas da preocupação masculina com a aparência. João Pessoa - PB: Dissertação de Mestrado apresentada à UFPB/CCHL, 2013.

GENTIL, P. Bases Científicas do Treinamento de Hipertrofia. Rio de Janeiro: Sprint, 2005.

GLANER, M.F. Validação cruzada de equações de impedância bioelétrica em homens. Rev. Bras. Cine. Des. Hum., V.7, n.1, p.5-11, 2005.

GRIEVE, G. A conceptual model of factors contributing to the development of muscle dysmorphia. Eating disorders, v.15, n.1, p.63-80, 2007.

GUARIN, H.P. Como problematizar La Educación Física desde La transicion Del concepto Del cuerpo al de corporeidad. Revista de Buenos Aires: Buenos Aires, 2002.

GUYTON, A.C; HALL, J.E. Tratado de fisiologia médica. 10. ed. Rio de Janeiro: Guanabara Koogan, 2002.

HARTGENS F., KUIPES H. Effects of androgenic-anabolic steroids in athletes. Sports Med., 2004.

HITZEROTH V. et al. Muscle dysmorphia: a South African sample. Psychiatry Clin Neurosci, 2001.

IRIART, J.; CHAVES, J..; ORLEANS, R. Culto ao corpo e o us de anabolizantes entre praticantes de musculação. Cad Saúde Pública, Rio de Janeiro, n.25, v.4, p.773-782, abr. 2009.

LANZT, C. D., RHEA, D. J.; CORNELIUS, A. E. Muscle Dysmorphia in elitelevel power lifters and body builders: a test of differences whitin a conceptual model. Journal of Strength and Conditioning Research, 16; 649-655, 2002.

LEONE, J. E.; SEDORY, E, J.; GRAY, K. A. Recognition and treatment of muscle dysmorphia and related body image disorders. Jornal of Atletic Training, v. 40, n. 4, p. 352-9, 2005.

MARTELL, C. A. Vigorexia: enfermedad o adaptación. Revista Digital de Deportes, v. 11, n. 99, 2006. 
MOTA, CRISTIANE; AGUIAR, EDUARDO. Dismorfia muscular: uma nova síndrome em praticantes de musculação. Revista Brasileira de Ciências da Saúde, 2011.

MUNOZ MARRÓN, E.; GÓMEZ CAMPELO, P. Adicción al deporte y déficit de atención selectiva. Encuentros en Psicología Social, v. 1, p. 74-76, 2003.

OLIVARDIA, R. Mirror, mirror on the wall, who's the largest of therm all? The features and phenomenology of muscle dysmorphia. Harvard Journal of Psychiatry, v. 9, p. 254294, 2001.

OLIVARDIA, R.; POPE JR; H.G.; HUDSON, J.J. Muscle dysmorphia in male weighlifters: A casecontrol stydy. The American Journal of Psychiarty, 2000.

PEREIRA, I. A. T. S. A vigorexia e os esteroides anabolizantes androgênicos em levantadores de pesos. Porto, Portugal: Dissertação de Licenciatura apresentada à FADEUP, 2009.

PINHEIRO, A. P.; GIUGLIANI, E. R. J. Quem são as crianças que se sentem gordas apesar de terem peso adequado? Jornal de Pediatria, v.82, n.3, p. 13-17, 2006.

POPE JR., H. G.; PHILLIPS, K. A.; OLIVARDIA, R. O complexo de Adônis: a obsessão masculina pelo corpo. Rio de Janeiro: Editora Campus, 2000.

POPE, H. G. J.; KARTZ, D. L.; HUDSON, J. I. Anorexia nervosa and "reverse anorexia" among 108 male bodybuilders. Comp Psychiatry., v. 34, p. 406-409, 1993.

POPE, H.G; Katz D. L. Bodybuilder's psychosis. Lancet, v. 1, n. 8537, p. 863-870, 1987.

PORTO, A. A.; LINS, R. G. Imagem corporal masculina e a mídia. Revista Digital EF Deportes, Buenos Aires, v. 14, n. 132, maio 2009.

QUADROS, T. M. B. et al. Imagem corporal em universitários: associação comestado nutricional e sexo. Revista Motriz, Rio Claro, v. 16, n. 1, p. 78-85, jan./mar., 2010.
RUSSO, R. Imagem Corporal: construção através da cultura do belo. Movimento \& Percepção, Espírito Santo de Pinhal, v.5, n.6, jan-jun, 2005.

SAIKALI, C. J.; SOUBHIA, C. S.; CORDAS, T. A. Imagem corporal nos transtornos alimentares. Rev. Psiq. Clin, São Paulo, v.31, n. 4, p. 164-166, 2004.

SANT ANA, A.L.; TEIXEIRA-ARROYO, C. Incidência da vigorexia em homens praticantes de musculação. Rev. Ed. Física UNIFABIPE, Bebedouro, SP, 2012.

SANTOS, M. A. A.; SANTOS, R. P. Uso de Suplementos Alimentares como forma de melhorar a performance nos programas de atividade física em academias de ginástica. Revista Paul.Educ., São Paulo, 16 (2): 174-85, Jul../Dez., 2002.

SCHILDER, P. A Imagem do Corpo: as energias construtivas da psique. São Paulo. Martins Fontes. 1999.

SEVERIANO, M. F. V.; RÊGO, M. O.; MONTEFUSCO, É, V. R. O corpo idealizado de consumo: paradoxos da hipermodernidade. Revista Mal-Estar e Subjetividade, v. 10, n. 1, p. 137-165, mar., 2010.

SILVA, P.; GOMES, E. Eternamente jovem: o corpo malhado, ficção televisual e imaginário. Pensar a prática 11/2, 2008. SOLER, PATRÍCIA et al. Vigorexia e níveis de dependência de exercício em frequentadores de academia e fisiculturistas.

VEGGI, A. B. et al. Índice de massa corporal, percepção do peso corporal e transtornos mentais comuns entre funcionários de uma universidade no Rio de Janeiro. Rev Bras de Psiquiatria., v. 26, n. 4, p. 242-247, 2004.

ZIMMERMANN, F. Indícios de vigorexia em adultos jovens praticantes de musculação em academias de Biguaçu-SC. Florianópolis, Dissertação de Bacharelado apresentado à UFSC, 2013.

YARYURA-TÓBIAS, J. A.; NEZIROGLU, F.; PÉREZ RIVERA, R. Obsesiones corporales. Buenos Aires: Editorial Polemos, 2003. 